\title{
Long-Range Interaction Potential of Open Shell Atoms with Neutral Molecules : Application to the Calculation of the Rate Constant for the $\mathrm{C}_{2} \mathrm{H}\left({ }^{2} \Sigma^{+}\right)+\mathrm{O}\left({ }^{3} \mathrm{P}\right)$ Reaction
}

\author{
Yuri Georgievskii and Stephen J. Klippenstein
}

Chemical Sciences and Engineering Division, Argonne National Laboratory, Argonne, IL 60439

\begin{abstract}
An accurate knowledge of the long-range interaction potential for the ground and first few excited electronic states is needed for quantitative prediction of the rate coefficients for astrochemical reactions at low temperatures. Some reactions important for astrochemical modeling include an open-shell atom as one of the fragments. Due to the interplay between the spin-orbit and quadrupole interactions such reactions require a special treatment. In this paper we derive the general expressions for the energy levels for such systems, apply them to the $\mathrm{C}_{2} \mathrm{H}\left({ }^{2} \Sigma^{+}\right)+\mathrm{O}\left({ }^{3} \mathrm{P}\right)$ reaction, and compare the results with ab initio calculations.
\end{abstract}

\section{Introduction}

Chemical modeling of the interstellar medium currently involves a large number of species $(>400)$ and reactions between them $(>4000)$ following the dramatic increase in the number of interstellar and circumstellar molecules detected via their rotational (sub)millimeter-wave spectra [Wakelam et al. (2010)]. Knowledge of the rate coefficients for these reactions is an important prerequisite of such modelling. Many of these reactions occur between highly reactive radical species under extremely low temperatures (i.e., $<50$ $\mathrm{K}$ and typically near $10 \mathrm{~K}$ ). Direct experimental measurements of the rate coefficients for such reactions are very difficult to obtain.

An important feature of many of these reactions is that they proceed via formation of a metastable reactive complex that may subsequently dissociate into different bimolecular products or be stabilized via radiative transitions and/or molecular collisions. A key complication arises from the fact that, due to the highly unsaturated nature of many of the species involved, not only the ground, but several excited electronic states with different spin may allow for formation a bound complex. However, not all of these electronic states contribute to the total rate since at low temperatures only barrierless entrance channels are accessible.

The master equation approach provides a standard procedure for calculating the rate coefficient for a complex-forming reaction [Miller \& Klippenstein (2006)]. In this approach all possible conformers of the reactive complex are properly characterized in terms of their densities of states and the transitions between them and to the reactants and products are characterized in terms of the corresponding numbers of states. Subsequent solution of the master equation provides the rate coefficients for reactions leading from reactants to all products. For the temperature range pertinent to astrochemical modelling, however, the situation is considerably simplified and the solution of the master equation is not required. Simple analysis shows that if there is a pathway from reactants to products with no barrier above the entrance channel the efficiency of the reaction is $100 \%$ and the 
corresponding rate coefficient is simply the rate for complex formation (i.e., the capture rate). On the other hand, if such a barrierless path does not exist, the rate coefficient is very small and can be neglected.

Thus, the rate coefficient calculation involves first the determination of the presence or absence of any barriers above the entrance channel for each of the possible electronic states follows by the evaluation of the capture rate for each reactive electronic state. The total rate coefficient is then the sum of these partial rate coefficients weighted by the appropriate Boltzmann factors.

The capture rate coefficient may be evaluated with variational transition state theory (VTST). We have recently developed the long-range transition state theory (LRTST), which is a special version of VTST adopted for low temperature applications [Georgievskii \& Klippenstein (2005)]. This theory is purely classical. Quantum mechanical treatments for non-reactive degrees of freedom are also available. Two essentially equivalent quantum approaches have been developed by Clary and coworkers [Clary (1990)], under the name of adiabatic capture centrifugal sudden approximation, and by Troe and coworkers [Troe (1997)], known as the statistical adiabatic channel model. The quantum treatment, however, is considerably more complicated and requires much more computational effort. Importantly, quantum effects are only important for temperatures of the order of and lower than the rotational constant of one of the fragments.

The long-range potential, which is needed for the capture rate coefficient calculation, is usually represented as a sum of a small number of terms representing interactions between major permanent multipole moments of the fragments and the $C_{6}$-term which represents induced dipole and dispersion interactions [Georgievskii \& Klippenstein (2005)]. The situation in which one of the fragments is an atom with an open electronic shell, such as $\mathrm{O}\left({ }^{3} \mathrm{P}\right)$ or $\mathrm{C}\left({ }^{3} \mathrm{P}\right)$, is more complicated. The major term in the long-range part of the potential for such atoms is the interaction of the atomic quadrupole, which depends on electronic angular momentum, with the multipole moments of the second fragment. Its interplay with the spin-orbit interaction of the atom requires a special treatment.

In this paper we derive general expressions for the energy levels of the atom in the ${ }^{3} \mathrm{P}$ electronic state in an arbitrary electric field. These expressions generalize the results of Gentry \& Giese (1977), who considered the interaction of an open-shell atom with an ion, and can be applied to the calculation of the interaction between an open-shell atom and a neutral molecule with the dipole, quadrupole, and higher multipole moments. We then apply the obtained expressions to calculate the rate coefficient for $\mathrm{C}_{2} \mathrm{H}\left({ }^{2} \Sigma^{+}\right)$ reacting with $\mathrm{O}\left({ }^{3} \mathrm{P}\right)$ and compare the result with corresponding results employing directly evaluated ab initio potentials. This reaction was found to be a key reaction in the recent review of interstellar chemistry by Wakelam et al. (2010).

\section{Potential Energy Surface}

The Hamiltonian of a quadrupole in an external electric field is given by [Landau \& Lifshitz (1991)],

$$
\hat{V}=\frac{1}{6} \frac{\partial^{2} \phi}{\partial x_{j} \partial x_{k}} \hat{Q}_{j k},
$$

where $\phi$ is the electric field potential and $\hat{Q}_{j k}$ is the operator for the quadrupole moment. The quadrupole moment, when averaged over all degrees of freedom but the direction of the angular momentum, may be expressed in terms of the angular momentum components 
as,

$$
\hat{Q}_{j k}=\frac{3 Q}{2 J(2 J-1)}\left(\hat{J}_{j} \hat{J}_{k}+\hat{J}_{k} \hat{J}_{j}-\frac{2}{3} J(J+1) \delta_{j k}\right),
$$

The Hamiltonian in Eq. 2.1 can be transformed to a more convenient form by appropriate rotation of the system of coordinates,

$$
\hat{V}=p_{x} \hat{Q}_{x x}+p_{y} \hat{Q}_{y y}+p_{z} \hat{Q}_{z z}
$$

where $p_{i}$ are the eigenvalues of the $\frac{1}{6} \frac{\partial^{2} \phi}{\partial x_{j} \partial x_{k}}$ matrix and the components of the quadrupole tensor are given in the new coordinate system. Note, that $p_{x}+p_{y}+p_{z}=0$. The important feature of this Hamiltonian is that it is invariant under the $\mathbf{D}_{\mathbf{2}}$ group, and the representations of that group can be used for classification of its energy levels.

For the dipole-quadrupole interaction the electric dipole potential is given by

$$
\phi(\mathbf{r})=\frac{\mathbf{d} \cdot \mathbf{r}}{r^{3}}
$$

where $\mathbf{d}$ is the dipole moment and $\mathbf{r}$ is the vector connecting the fragments. The second derivative of this potential is given by

$$
\frac{\partial^{2} \phi}{\partial x_{j} \partial x_{k}}=15 \frac{\mathbf{d} \cdot \mathbf{r}}{r^{7}} x_{j} x_{k}-3 \frac{d_{j} x_{k}+d_{k} x_{j}}{r^{5}}-3 \frac{\mathbf{d} \cdot \mathbf{r}}{r^{5}} \delta_{k j} .
$$

Without restriction of generality one can assume that $\mathbf{r}$ is directed along the z-axis and d belongs to the zx-plane. Then, the following expressions for the $p_{i}$ parameters are obtained with rotation around the y-axis,

$$
\left(\begin{array}{l}
p_{x} \\
p_{y} \\
p_{z}
\end{array}\right)=\frac{d}{2 r^{4}}\left(\begin{array}{c}
1 / 2 \cos \theta-\sqrt{1+5 / 4 \cos ^{2} \theta} \\
-\cos \theta \\
1 / 2 \cos \theta+\sqrt{1+5 / 4 \cos ^{2} \theta}
\end{array}\right),
$$

where $\theta$ is the angle between the $\mathbf{d}$ and $\mathbf{r}$ directions. It is worth noting that $p_{x}<p_{y}<p_{z}$

We will consider first the strong and the weak spin-orbit coupling limits and then the general case.

\subsection{Strong spin-orbit coupling limit}

This case corresponds to low temperatures where the relevant separations between the fragments a large. In this limit the total angular momentum is a good quantum number and each spin-orbit manifold should be considered separately. The spin-orbit splitting at infinite separation provides the reference energy for each manifold. In this limit one should understand under $\hat{\mathbf{J}}$ in Eq. 2.2 the total electronic angular momentum $\hat{\mathbf{J}}=\hat{\mathbf{L}}+\hat{\mathbf{S}}$ and use the effective quadrupole moment $Q_{J}$ instead of $Q$ in Eq. 2.2. The value of $Q_{J}$ depends on $J$.

\section{$J=2$ quintet.}

In this spin-orbit multiplet $Q_{J}=Q$. Under the $\mathbf{D}_{2}$ group it is split into two A representations, $\left|A^{\prime}\right\rangle \equiv|0\rangle$ and $\left|A^{\prime \prime}\right\rangle \equiv \sqrt{1 / 2}(|2\rangle+|-2\rangle)$, and three B representations, with one each of the $B_{x}, \sqrt{1 / 2}(|1\rangle+|-1\rangle), B_{y}, \sqrt{1 / 2}(|1\rangle-|-1\rangle)$, and $B_{z}$ representations, $\sqrt{1 / 2}(|2\rangle-|-2\rangle)$. Here by $B_{i}$ we denote the representation which is unchanged under $C_{2}^{i}$ transformation, $\mathrm{A}$ is the totally symmetric representation, and $\left|M_{J}\right\rangle$ is a state with a given $\hat{J}_{z}$ value. In the following table we show the $\hat{J}_{x}^{2}, \hat{J}_{y}^{2}$, and $\hat{J}_{z}^{2}$ matrices and the 


$$
\mathrm{C}_{2} \mathrm{H}+\mathrm{O}
$$

Hamiltonian eigenvalues in each of these subspaces:

\begin{tabular}{|c|c|c|c|c|c|}
\hline$R$ & |\rangle & $\hat{J}_{x}^{2}-2$ & $\hat{J}_{y}^{2}-2$ & $\hat{J}_{z}^{2}-2$ & $E$ \\
\hline$A$ & $\begin{array}{c}|0\rangle \\
\sqrt{\frac{1}{2}}(|2\rangle+|-2\rangle)\end{array}$ & $\left(\begin{array}{cc}1 & \sqrt{3} \\
\sqrt{3} & -1\end{array}\right)$ & $\left(\begin{array}{cc}1 & -\sqrt{3} \\
-\sqrt{3} & -1\end{array}\right)$ & $\left(\begin{array}{cc}-2 & 0 \\
0 & 2\end{array}\right)$ & $\begin{array}{c} \pm \sqrt{\frac{3}{2} Q \times} \\
\sqrt{p_{x}^{2}+p_{y}^{2}+p_{z}^{2}}\end{array}$ \\
\hline$B_{x}$ & $\sqrt{\frac{1}{2}}(|1\rangle+|-1\rangle)$ & 2 & -1 & -1 & $\frac{3}{2} Q p_{x}$ \\
\hline$B_{y}$ & $\sqrt{\frac{1}{2}}(|1\rangle-|-1\rangle)$ & -1 & 2 & -1 & $\frac{3}{2} Q p_{y}$ \\
\hline$B_{z}$ & $\sqrt{\frac{1}{2}}(|2\rangle-|-2\rangle)$ & -1 & -1 & 2 & $\frac{3}{2} Q p_{z}$ \\
\hline
\end{tabular}

For the dipole-quadrupole interaction it gives the following energies,

\begin{tabular}{|c|c|}
\hline$R$ & $E$ \\
\hline$A$ & $\pm \frac{\sqrt{3}}{2} V_{0} \sqrt{2 \cos ^{2} \theta+1}$ \\
\hline$B_{x}$ & $\frac{3}{8} V_{0}\left(\cos \theta-\sqrt{5 \cos ^{2} \theta+4}\right)$ \\
\hline$B_{y}$ & $-\frac{3}{4} V_{0} \cos \theta$ \\
\hline$B_{z}$ & $\frac{3}{8} V_{0}\left(\cos \theta+\sqrt{5 \cos ^{2} \theta+4}\right)$ \\
\hline
\end{tabular}

where we have used the notation $V_{0}=d Q / r^{4}$ for the strength of the dipole-quadrupole interaction.

$J=1$ triplet.

In this spin-orbit multiplet $Q_{J}=-Q / 2$. Under the $\mathbf{D}_{2}$ group it is split into three, with one each of the $B_{x}, B_{y}$, and $B_{z}$ representations. The corresponding states $|x\rangle,|y\rangle$, and $|z\rangle$ are defined by having zero projection of the angular momentum on the corresponding axis, $\hat{J}_{i}=0$.

\begin{tabular}{|c|c|c|c|c|}
\hline$R||\rangle$ & $\hat{J}_{x}^{2}-\frac{2}{3}$ & $\hat{J}_{y}^{2}-\frac{2}{3}$ & $\hat{J}_{z}^{2}-\frac{2}{3}$ & $E$ \\
\hline$\left.B_{x}|| x\right\rangle$ & $-2 / 3$ & $1 / 3$ & $1 / 3$ & $\frac{3}{2} Q p_{x}$ \\
\hline$\left.B_{y}|| y\right\rangle$ & $1 / 3$ & $-2 / 3$ & $1 / 3$ & $\frac{3}{2} Q p_{y}$ \\
\hline$\left.B_{z}|| z\right\rangle$ & $1 / 3$ & $1 / 3$ & $-2 / 3$ & $\frac{3}{2} Q p_{z}$ \\
\hline
\end{tabular}

\subsection{Strong quadrupole limit}

In this case one can neglect the spin-orbit interaction and consider only the orbital part of the wave function. The three-dimensional orbital space is split into three one-dimensional representations, $|i\rangle, i=x, y, z$, which denote the states with zero projection of the angular 
momentum on the $i$-th axis $L_{i}=0$,

\begin{tabular}{|c|c|c|c|c|c|}
\hline$R$ & |\rangle & $\hat{L}_{x}^{2}-\frac{2}{3}$ & $\hat{L}_{y}^{2}-\frac{2}{3}$ & $\hat{L}_{z}^{2}-\frac{2}{3}$ & $E$ \\
\hline$\left.B_{x}|| x\right\rangle$ & $-2 / 3$ & $1 / 3$ & $1 / 3$ & $-3 Q p_{x}$ \\
\hline$\left.B_{y}|| y\right\rangle$ & $1 / 3$ & $-2 / 3$ & $1 / 3$ & $-3 Q p_{y}$ \\
\hline$\left.B_{z}|| z\right\rangle$ & $1 / 3$ & $1 / 3$ & $-2 / 3$ & $-3 Q p_{z}$ \\
\hline
\end{tabular}

For the dipole-quadrupole interaction it gives the following energies,

\begin{tabular}{|c|c|}
\hline$R$ & $E$ \\
\hline$B_{x}$ & $\frac{3}{4} V_{0}\left(-\cos \theta+\sqrt{5 \cos ^{2} \theta+4}\right)$ \\
\hline$B_{y}$ & $\frac{3}{2} V_{0} \cos \theta$ \\
\hline$B_{z}$ & $\frac{3}{4} V_{0}\left(-\cos \theta-\sqrt{5 \cos ^{2} \theta+4}\right)$ \\
\hline
\end{tabular}

The last expression shows that there are six attractive and three repulsive potential energy surfaces in the dipole-quadrupole approximation.

\subsection{General case}

The total electronic Hamiltonian with the spin-orbit coupling is

$$
\hat{H}=\hat{V}_{d Q}-A \hat{\mathbf{S}} \cdot \hat{\mathbf{L}} \text {. }
$$

We have deliberately chosen the minus sign in front of the spin-orbit interaction term to stress that $J=2$ is the ground spin-orbit multiplet for oxygen atom. Under the $\mathbf{D}_{2}$ group the nine-dimensional spin-orbit space of this Hamiltonian is split into the three $A$ representations and two of each $B_{x}, B_{y}$, and $B_{z}$ representation. In the following table the diagonal components of the quadrupole operator and the full $\mathbf{S} \cdot \mathbf{L}$ matrix are shown in each of these subspaces in the appropriate basis.

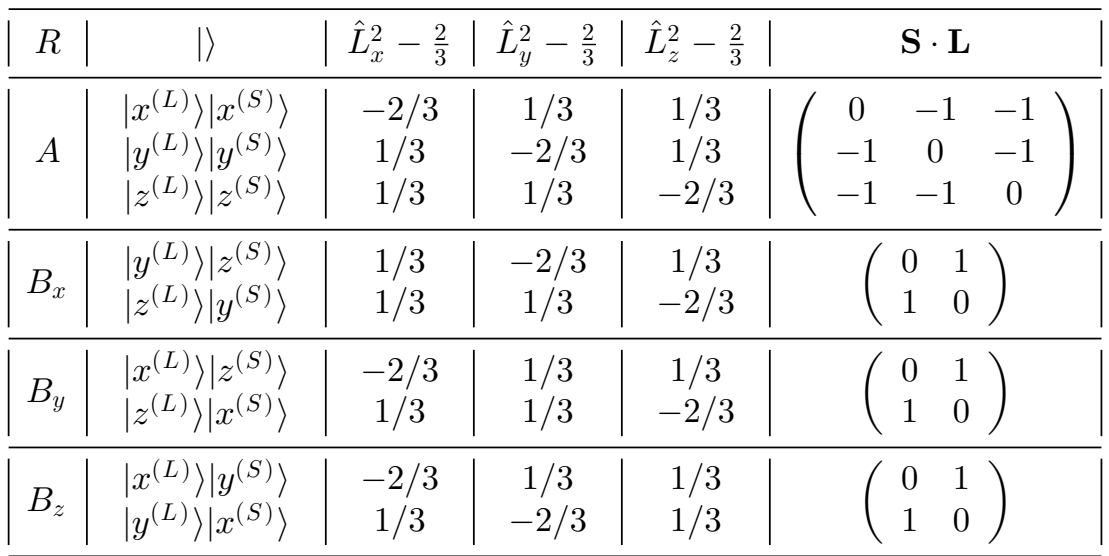

Using these expressions one can explicitly write down the expressions for the energies. For the $B_{x}$ subspace one obtains

$$
E_{1,2}^{(x)}=\frac{3}{2} Q p_{x} \pm \sqrt{A^{2}+\frac{9}{4} Q^{2}\left(p_{z}-p_{y}\right)^{2}} .
$$




$$
\mathrm{C}_{2} \mathrm{H}+\mathrm{O}
$$

The energies for the $B_{y}$ and $B_{z}$ subspaces are obtained from Eq. 2.14 by permutation of indices. In the case of the $\mathrm{A}$ representation the energies are the eigenvalues of the following Hamiltonian,

$$
\left(\begin{array}{ccc}
-3 Q p_{x} & A & A \\
A & -3 Q p_{y} & A \\
A & A & -3 Q p_{z}
\end{array}\right) .
$$

We explicitly consider the important specific case, $p_{x}=p_{y}=-p_{z} / 2$. In this case Eq. 2.15 has a simple solution,

$$
\begin{aligned}
& E_{1}^{(a)}=-A+\frac{3}{2} Q p, \\
& E_{2,3}^{(a)}=-A+\frac{3}{2}\left(A-\frac{Q p}{2} \pm \sqrt{A^{2}+A Q p+\frac{9}{4} Q^{2} p^{2}}\right),
\end{aligned}
$$

where $p \equiv p_{z}$.

In the general case, instead of writing the solution of Eq. 2.15 explicitly, which is rather cumbersome, we show how the energies correlate with the strong quadrupole and strong spin-orbit coupling limits. Assuming that $p_{x}<p_{y}<p_{z}$ one obtains the following correlation table,

\begin{tabular}{|c|c|c|c|c|}
\hline$R$ & $\mathrm{dQ}-$ limit & $T$ & SL - limit & $T$ \\
\hline \multirow{3}{*}{$A$} & $-3 Q p_{x}+\frac{A^{2}}{3 Q}\left(\frac{1}{p_{y}-p_{x}}+\frac{1}{p_{z}-p_{x}}\right)$ & $R$ & 1 & $?$ \\
& $-3 Q p_{y}+\frac{A^{2}}{3 Q}\left(\frac{1}{p_{x}-p_{y}}+\frac{1}{p_{z}-p_{y}}\right)$ & $A$ & $-A+\sqrt{\frac{3}{2}} Q \sqrt{p_{x}^{2}+p_{y}^{2}+p_{z}^{2}}$ & $?$ \\
& $-3 Q p_{z}+\frac{A^{2}}{3 Q}\left(\frac{1}{p_{x}-p_{z}}+\frac{1}{p_{y}-p_{z}}\right)$ & $A$ & $-A-\sqrt{\frac{3}{2}} Q \sqrt{p_{x}^{2}+p_{y}^{2}+p_{z}^{2}}$ & $A$ \\
\hline \multirow{2}{*}{$B_{x}$} & $-3 Q p_{y}+\frac{A^{2}}{3 Q} \frac{1}{p_{z}-p_{y}}$ & $A$ & $A+\frac{3}{2} Q p_{x}$ & $A$ \\
& $-3 Q p_{z}+\frac{A^{2}}{3 Q} \frac{1}{p_{y}-p_{z}}$ & $A$ & $-A+\frac{3}{2} Q p_{x}$ & $A$ \\
\hline \multirow{2}{*}{$B_{y}$} & $-3 Q p_{x}+\frac{A^{2}}{3 Q} \frac{1}{p_{z}-p_{x}}$ & $R$ & $A+\frac{3}{2} Q p_{y}$ & $A$ \\
\hline \multirow{2}{*}{$B_{z}$} & $-3 Q p_{z}+\frac{A^{2}}{3 Q} \frac{1}{p_{x}-p_{z}}$ & $A$ & $-A+\frac{3}{2} Q p_{y}$ & $A$ \\
\hline & $-3 Q p_{x}+\frac{A^{2}}{3 Q} \frac{1}{p_{y}-p_{x}}$ & $R$ & $A+\frac{3}{2} Q p_{z}$ & $R$ \\
\hline
\end{tabular}

From this table one can see that there are two surfaces, $A^{(2)}$ and $B_{z}^{(2)}$, which should have barriers (repulsive behavior at large separation changing to attraction at smaller distances) and one surface with a well, $B_{y}^{(1)}$ (long-range attraction changes to repulsion at shorter separations). In Fig. 1 a diagram is shown that correlates the energy levels for the dipole-quadrupole interaction in the strong and weak spin-orbit limits.

\section{3. $\mathrm{C}_{2} \mathrm{H}\left({ }^{2} \Sigma^{+}\right)+\mathrm{O}\left({ }^{3} \mathrm{P}\right)$ Reaction}

If one neglects for a moment the spin-orbit interaction, there are three triply-degenerate electronic states for $\mathrm{O}\left({ }^{3} \mathrm{P}\right)$ and one doubly-degenerate electronic state for $\mathrm{C}_{2} \mathrm{H}\left({ }^{2} \Sigma^{+}\right)$. Altogether it gives eighteen electronic surfaces for the reactive complex. When strongly coupled, it provides three doublet $(S=1 / 2)$ and three quartet $(S=3 / 2)$ electronic PESs. 

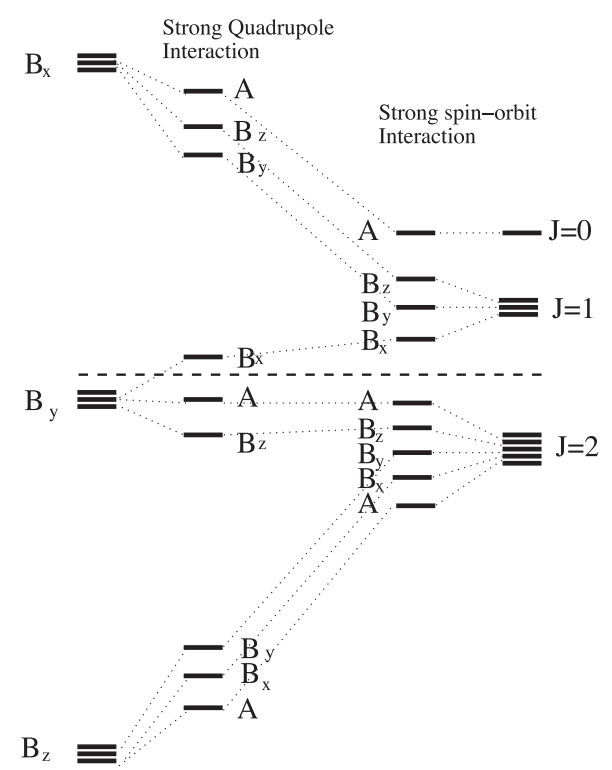

Figure 1. The correlation diagram for the energy levels of the quadrupole in an external electric field.

To figure out which of these surfaces are attractive we have performed minimum energy path (constrained optimization) calculations for all three doublet and three quartet states. We evaluate these interactions with the CASPT2 method employing nine-electron, eight-orbital $(9 \mathrm{e}, 8 \mathrm{o})$ active space consisting of three p-orbitals for $\mathrm{O}(4 \mathrm{e}, 3 \mathrm{o})$ and two $\pi$, two $\pi^{*}$ (full $\pi, \pi^{*}$ space), and one radical $\sigma$ orbitals for $\mathrm{C}_{2} \mathrm{H}(5 \mathrm{e}, 5 \mathrm{o})$. These CASPT2 calculations employ Dunning's correlation-consistent, polarized valence, double- $\zeta$ basis set [Dunning (1989)] and were done using the formalism of Celani and Werner [Celani \& Werner (2000)] as implemented in the MOLPRO quantum chemistry package [Molpro (2010)].

Our calculations show that there are two attractive doublet potential energy surfaces which correlate with the two $\mathrm{CO}+\mathrm{CH}\left({ }^{2} \Pi\right)$ product states and one attractive quartet surface which correlates with the $\mathrm{CO}+\mathrm{CH}\left({ }^{4} \Sigma^{-}\right)$products. All other PESs have potential barriers higher than the reactants and can be neglected.

Thus, there are altogether eight spin-orbital states of the reactive complex which contribute to the total rate constant. In the considered analytical approximation for each spin-orbital state of the oxygen atom in the electric field of the $\mathrm{C}_{2} \mathrm{H}$ fragment an additional degeneracy of two should be added because of the spin degeneracy of the $\mathrm{C}_{2} \mathrm{H}\left({ }^{2} \Sigma^{+}\right)$ fragment itself. Thus, the capture rate coefficients should be calculated only for four lowest spin-orbital states of the oxygen atom in the electric field of the $\mathrm{C}_{2} \mathrm{H}$ fragment. These four states correlate with four $J=2$ multiplet states of the oxygen atom at infinite separation.

The important feature of the $\mathrm{O}\left({ }^{3} \mathrm{P}\right)+\mathrm{C}_{2} \mathrm{H}\left({ }^{2} \Sigma^{+}\right)$reaction is that the dipole moment $d$ of the $\mathrm{C}_{2} \mathrm{H}$ fragment is relatively small $(d \simeq 0.31$ a.u.) while the quadrupole moment $Q \equiv Q_{z z}$ is relatively large $(Q \simeq 7.1$ a.u.). Thus, to correctly reproduce $a b$ initio data in addition to the dipole-quadrupole interaction one should at least include the quadrupolequadrupole interaction term.

To compare the analytical and ab initio PESs we consider the linear configuration with the oxygen atom approaching the $\mathrm{C}_{2} \mathrm{H}$ fragment from both the carbon and hydrogen 


$$
\mathrm{C}_{2} \mathrm{H}+\mathrm{O}
$$

sides. In this situation $p_{x}=p_{y}=-p_{z} / 2$ and Eqs. 2.16 and 2.17 are applicable. The $a b$ initio calculations have been performed at the CASPT2(5e,4o)/cc-pvqz level. In Fig. 2 both the four lowest analytical and ab initio PESs are shown for the oxygen atom on the carbon side of the $\mathrm{C}_{2} \mathrm{H}$ fragment. The $C_{6}$ dispersion coefficient was approximated

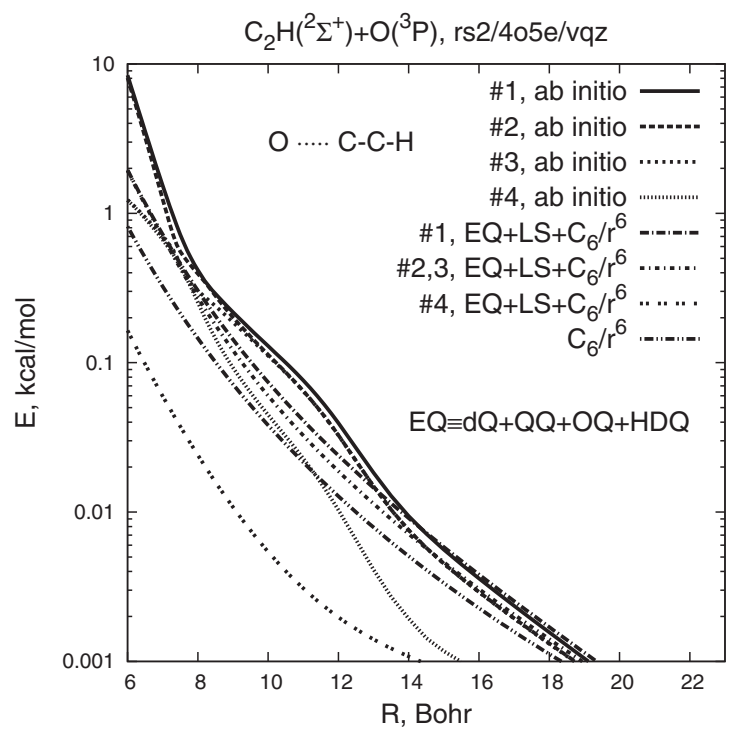

Figure 2. The four lowest potential energy surfaces for the $\mathrm{O}\left({ }^{3} \mathrm{P}\right)+\mathrm{C}_{2} \mathrm{H}\left({ }^{2} \Sigma^{+}\right)$reaction with the oxygen approaching $\mathrm{C}_{2} \mathrm{H}$ on the carbon side.

according to the standard formula [Hirschfelder, Curtiss, \& Bird (1967)],

$$
C_{6}=1.5 \alpha_{1} \alpha_{2} I_{1} I_{2} /\left(I_{1}+I_{2}\right) C F,
$$

where $\alpha_{i}, I_{i}$ are the polarizability and the ionization energy of the $i$-th fragment, and the correction factor $C F=2.3$ was chosen to better fit $a b$ initio energies at large separation distances $R$.

In Fig. 3 both the four lowest analytical and ab initio PESs are shown for the oxygen atom on the hydrogen side of the $\mathrm{C}_{2} \mathrm{H}$ fragment.

While at larger distances, $R>14 \mathrm{Bohr}$, the agreement is almost perfect, in the intermediate distance range, $8<R<12$, Bohr the analytical and ab initio energies may differ by up to a factor of two. We note that this difference strongly depends on the basis set. One of the reasons for this difference may be related to the basis set superposition error in the $a b$ initio calculations. At smaller distances, $R<8$ Bohr, chemical interaction becomes the dominant one and should be included in the energy estimates.

To calculate the $\mathrm{C}_{2} \mathrm{H}+\mathrm{O}$ reaction rate coefficients for each electronic PES the variable reaction coordinate transition state theory code [Georgievskii \& Klippenstein (2003)], which was developed to treat the radical-radical association reactions, has been used. $A b$ initio energies were calculated directly "on the fly" using cc-pvqz basis set. The non-diagonal spin-orbit matrix elements were calculated on the MCSCF $(4 \mathrm{e}, 3 \mathrm{o})$ level and the diagonal energies on the CASPT2 $(4 \mathrm{e}, 3 \mathrm{o})$ level. The analytical calculations included dipole-quadrupole, quadrupole-quadrupole, dipole-induced dipole, and dispersion interaction terms. In Fig. 4 the partial rate coefficients calculated both with analytical and $a b$ initio methods are shown for different electronic surfaces as functions of temperature as well as the total rate coefficient. One can see that the difference between the analytical 


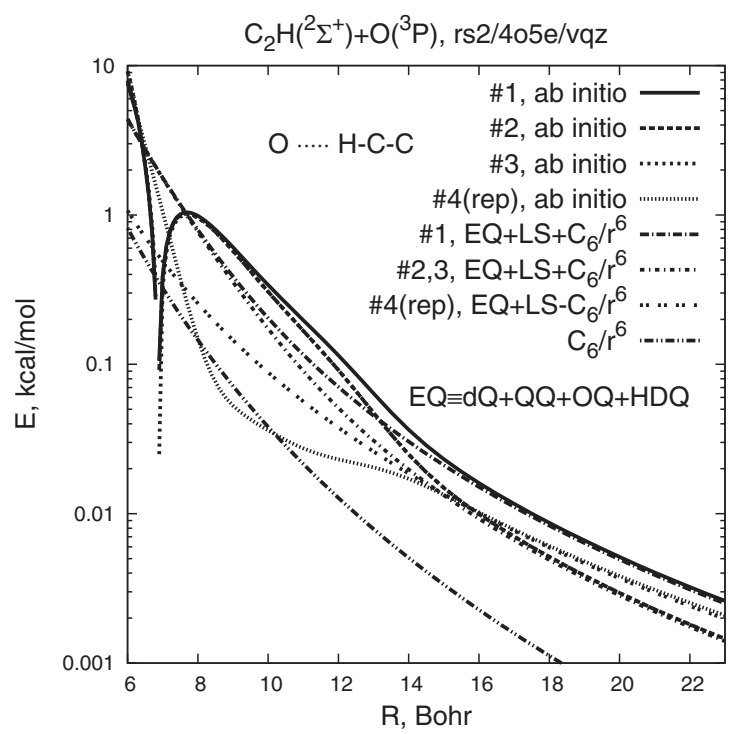

Figure 3. The four lowest potential energy surfaces for the $\mathrm{O}\left({ }^{3} \mathrm{P}\right)+\mathrm{C}_{2} \mathrm{H}\left({ }^{2} \Sigma^{+}\right)$reaction with the oxygen approaching $\mathrm{C}_{2} \mathrm{H}$ on the hydrogen side.

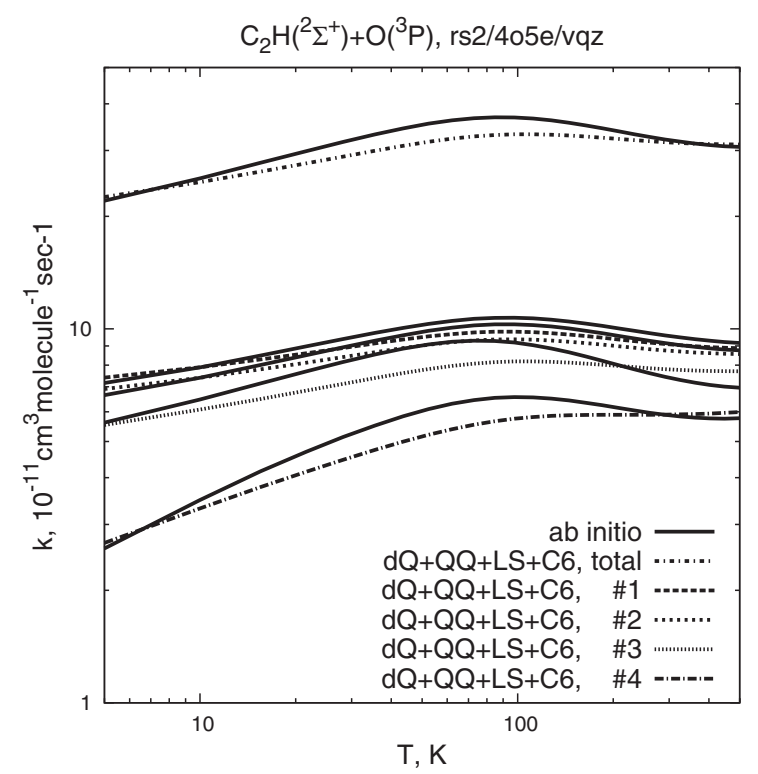

Figure 4. The capture rate coefficients for the $\mathrm{O}\left({ }^{3} \mathrm{P}\right)+\mathrm{C}_{2} \mathrm{H}\left({ }^{2} \Sigma^{+}\right)$reaction as functions of temperature.

and $a b$ initio potential calculations does not exceed $10 \%$ in the whole temperature range considered.

\section{Conclusions}

Analytical expressions for the energy levels of an open-shell ${ }^{3} \mathrm{P}$ atom in an external electric field have been derived. The obtained expressions provide a simple and valuable means to calculate the long-range part of the interaction potential between an open-shell 


$$
\mathrm{C}_{2} \mathrm{H}+\mathrm{O}
$$

${ }^{3} \mathrm{P}$ atom and a neutral molecule. They have been applied to the $\mathrm{O}\left({ }^{3} \mathrm{P}\right)+\mathrm{C}_{2} \mathrm{H}\left({ }^{2} \Sigma^{+}\right)$reaction. The comparison between the rate coefficients obtained with the use of the analytical and $a b$ initio potentials show very good agreement in the whole considered temperature range $(5-500 \mathrm{~K})$. This good agreement suggests that the theoretical predictions are of high accuracy.

\section{Acknowledgments}

This work was supported by NASA's Planetary Atmospheres Program through grant NNH09AK24I. Computational support was provided by the U. S. Department of Energy, Office of Basic Energy Sciences, Division of Chemical Sciences, Geosciences, and Biosciences under DOE Contract Number DE-AC02-06CH11357. We thank Larry Harding for valuable discussions.

\section{References}

Wakelam, V., Smith, I. W. M., Herbst, E., Troe, J., Geppert, W., Linnartz, H., Öberg, K., Roueff, E., Agúndez, M., Pernot, P., Cuppen, H. M., Loison, J. C., \& Talbi, D. Space Sci. Rev. 2010.

Miller, J. A. \& Klippenstein, S. J. J. Phys. Chem. A 2006, 110, 10528.

Georgievskii, Y. \& Klippenstein, S. J. J. Chem. Phys. 2005, 122, 194103.

Clary, D. C. Ann. Rev. Phys. Chem. 1990, 41, 61.

Troe, J. Adv. Chem. Phys. 1997, 101, 819.

Gentry, W. R. \& Giese, C. F. J. Chem. Phys. 1977, 67, 2355.

Landau, L. D. \& Lifshitz, E. M. Quantum Mechanics; Pergamon Press: New York, 1991.

T. H. Dunning, J. J. Chem. Phys. 1989, 90, 1007.

Celani, P. \& Werner, H.-J. J. Chem. Phys. 2000, 112, 5546.

Molpro, version 2010.1, a package of ab initio programs. Werner, H.-J., Knowles, P. J., Manby, F. R., Schütz, M., Celani, P., Knizia, G., Korona, T., Lindh, R., Mitrushenkov, A., Rauhut, G., Adler, T. B., Amos, R. D., Bernhardsson, A., Berning, A., Cooper, D. L., Deegan, M. J. O., Dobbyn, A. J., Eckert, F., Goll, E., Hampel, C., Hesselmann, A., Hetzer, G., Hrenar, T., Jansen, G., Köppl, C., Liu, Y., Lloyd, A. W., Mata, R. A., May, A. J., McNicholas, S. J., Meyer, W., Mura, M. E., Nicklass, A., Palmieri, P., Pflüger, K., Pitzer, R., Reiher, M., Shiozaki, T., Stoll, H., Stone, A. J., Tarroni, R., Thorsteinsson, T., Wang, M., \& Wolf, A. 2010.

Hirschfelder, J. O., Curtiss, C. F., \& Bird, R. B. Molecular Theory of Gases and Liquids; John Wiley \& Sons: New York, 1967.

Georgievskii, Y. \& Klippenstein, S. J. J. Chem. Phys. 2003, 118, 5442.

\section{Discussion}

Stephan Schlemmer: What is the influence of diabatic coupling on the results of calculation of $k(T)$, especially at low temperatures?

Stephen J. Klippenstein: The statistical treatment of the electronic states gives some indication of the maximum effect of the diabatic couplings. Sample calculations suggest that these effects are typically quite small: a few $\%$ or less.

E. F. VAN DishoECK: Thanks for calculating all of these astrophysically interesting reactions. In interstellar space, the populations of the fine structure leves of, say, $\mathrm{O}\left({ }^{3} \mathrm{P}\right)$ or $\mathrm{C}\left({ }^{3} \mathrm{P}\right)$ are often subthermal. It would be very useful for astrochemists to "pull out" these state-specific rate coefficients explicitly from your calculation. 
Stephen J. KLippenstein: That can readily be done from our electronic state specific results and we will do so in the future.

IAN SMITH: In radical-radical reactions surfaces of several spin multiplicities correlate with the reactants. I would expect the surfaces of low multiplicity (where electrons have "paired up") to have no barrier. I am less sure about the surfaces of higher multiplicities. Have you checked to all that these surfaces have no barrier?

Stephen J. Klippenstein: Yes, we have and this is one of the important steps in the rate constant calculation. Typically we found that for reactions with either $\mathrm{O}\left({ }^{3} \mathrm{P}\right)$ or $\mathrm{C}\left({ }^{3} \mathrm{P}\right)$ and a radical species in the doublet $(S=1 / 2)$ electronic state, the two doublet electronic surfaces and one quartet surface are barrierless while all other surfaces have significant barriers and, therefore, are not reactive. 